\title{
Análisis de la dolarización y sus efectos sobre la inversión extranjera directa en Ecuador. Periodo 2000 - 2016
}

\author{
Eduardo Zurita -Moreano', Gabriela González-Bautista², Eugenia Borja- Lombeida³
}

Fecha de recepción: 13 de Septiembre 2017

Fecha de aceptación: 11 de Diciembre 2017

\section{Resumen}

El presente artículo se enfoca en las consecuencias que ha tenido la implementación de la dolarización en la economía ecuatoriana y a su vez, las repercusiones de ésta sobre las decisiones de los inversionistas extranjeros, la metodología de investigación utilizada para el análisis de la dolarización se realizó a través de las variables macroeconómicas más importantes con relación a la inversión extranjera directa, los resultados de la estimación del modelo econométrico se los obtuvo mediante una regresión lineal múltiple, estimada por el método de mínimos cuadrados ordinarios. Se demuestra que la dolarización, medida a través de los indicadores: inversión en electricidad, índice de precios al consumidor, salario básico unificado, gasto en educación y PIB tienen una relación directa con la inversión extranjera directa.

Palabras clave: Dolarización, inversión extranjera directa, salario básico unificado, gasto en educación, producto interno bruto.

\begin{abstract}
The present article focuses on the consequences of the dollarization in the ecuadorian economy and the decisions of foreign investors, the research methodology used to analyze the dollarization was done through the most important macroeconomic variables in relation to foreign direct investment, the results of the estimation of the econometric model were obtained by a multiple linear regression, estimated by the method of ordinary least squares. It is shown that dollarization, measured through the indicators: investment in electricity, consumer price index, unified basic salary, education expenditure and GDP have a direct relationship with foreign direct investment.
\end{abstract}

Keywords: Dollarization, foreign direct investment, unified basic salary, education expenditure; gross domestic product.

\section{Introducción}

A lo largo del tiempo Ecuador ha asumido diversos cambios, uno de los más importantes fue el cambio de la moneda propia, el Sucre, por el dólar estadounidense, medida adoptada debido a la profunda crisis económica y del sistema financiero por la que atravesaba el país, sumado a esto la elevada tasa de inflación a finales del año 1999. La idea de esta investigación es determinar los efectos que ha tenido la dolarización en la Inversión Extranjera Directa a partir del año 2000, periodo en que se adopta la nueva medida económica. Cabe mencionar que las empresas extranjeras a más de buscar países con estabilidad económica y menor incertidumbre de su situación macroeconómica futura, requieren salarios inferiores al de sus países, aumento en la calidad de educación de la fuerza laboral, para mejorar la productividad, así como también, países con calidad de infraestructura.

La investigación se desarrolló bajo el contexto del Paradigma Ecléctico de Dunning, que según Bilbao \& Longás (2009) plantean que la internacionalización de una empresa obedece a la búsqueda de recursos, mercados, eficiencia productiva y de activos estratégicos, sobre todo la rentabilidad a largo plazo. Para establecer la relación entre la dolarización y la Inversión Extranjera Directa; las variables independientes consideradas fueron: inversión en la electricidad, índice de precios al consumidor, salario básico unificado, gasto anual en educación y producto interno bruto; la inversión extranjera directa como la variable dependiente.

\section{Metodología}

Varios analistas económicos manifiestan que el propósito de la dolarización consiste en mantener la estabilidad de los precios con crecimiento económico y pleno empleo. Por lo tanto, para Rosillo (2011), son varios factores que determinan la orientación de los flujos de IED de un país o región hacia otro, tales como los factores institucionales, políticos y económicos que intervienen directamente en la decisión del inversionista, el cual menciona los siguientes: tamaño de mercado, riesgo, costos laborales, estabilidad macroeconómica, políticas comerciales, intensidad y disponibilidad de los factores productivos, otras ventajas comparativas(costos de transporte, políticas públicas, incentivos fiscales y exenciones de impuestos).

La investigación se realizó basada en el modelo de John Dunning determinado marco OLI o paradigma ecléctico. Para la elección de las variables a incluir, se tomaron consideraciones sobre la disponibilidad de la información y las más representativas, en base a la teoría antes citada sobre el modelo OLI:

$\ln Y_{i}=\hat{\beta}_{0}+\hat{\beta}_{1} * \ln X_{1}+\hat{\beta}_{2} * \ln X_{2}+\hat{\beta}_{3} * \ln X_{3}+\hat{\beta}_{4} * \ln X_{4}+\hat{\beta}_{5} * \ln X_{5}+u_{i}$

En donde:

$Y=$ Inversión Extranjera Directa

$\mathrm{X}_{1}=$ ELEC $=$ Inversión eléctrica (Usd)

$\mathrm{X}_{2}=$ IPC $=$ Índice de precios al consumidor (Usd)

$\mathrm{X}_{3}=\mathrm{SBU}=$ Salario básico unificado (Usd)

$\mathbf{X}_{4}=$ EDU $=$ Inversión pública en educación (Usd)

$\mathrm{X}_{5}=\mathrm{PIB}=$ Producto Interno Bruto real (Usd)

$\beta_{0}=$ Valor constante $\mathrm{o}$ intercepto del modelo

u= Término de perturbación estocástico

Reemplazando la expresión se obtiene la siguiente:

$\operatorname{lnIED_{i}}=\hat{\beta}_{0}+\hat{\beta}_{1} * \ln \mathrm{ELEC}+\hat{\beta}_{2} * \ln I P C_{2}+\hat{\beta}_{3} * \ln S B U_{3}+\hat{\beta}_{4} * \ln E D U_{4}+\hat{\beta}_{5} * \ln P I B_{5}+u_{i}$

Donde, los parámetros ( $\beta_{-}^{\wedge} 0, \beta_{-}^{\wedge} 1, \beta^{\wedge} \_, \beta_{-}^{\wedge} 3, \beta_{-}^{\wedge} 4, \beta_{-}^{\wedge}$ ) son los coeficientes de regresión que miden las elasticidades parciales de las variables explicativas. La presente investigación se desarrolló dentro del modelo de Regresión Lineal Múltiple, estimado por el método de Mínimos Cuadrados Ordinarios (MCO) y así establecer la relación entre la dolarización y la Inversión Extranjera Directa.

\section{Resultados}

En 1884 en el Ecuador circulaba una moneda propia denominada sucre, la misma que al transcurrir 116 años fue legalmente sustituida por el dólar estadounidense. En 1990 el 99,9\% de los depósitos de ahorro y plazo fijo, así como el 92, 5\% del cuasi dinero en la economía se encontraban en sucres, cifras que variaron para el año de 1996 pasando a un $23,8 \%$ y $28 \%$ respectivamente. (Emanuel, 2015, p. 15)

Lo que se produjo a finales de 1990 fue una desencadenada fiebre por el dólar, generado por la desconfianza del sucre, por lo que la moneda nacional sufre una fuerte devaluación que pasó de 5.000 sucres por dólar en 1995 a 25.000 sucres por dólar en el 2000, al anunciar la dolarización el 9 de enero del mismo año, la economía ecuatoriana ya se hallaba dolarizaba informalmente en un 60\%. Para Larrea (2010), los principales efectos que se derivaron de la dolarización fueron, una caida de la inflación, un mejoramiento en el nivel de vida, estabilidad macroeconómica, y apertura de mercados. En lo referente al Producto Interno Bruto entre el periodo 1995 - 1999 según datos del Banco Central del Ecuador presentó un incremento del 1,2\%, en cambio, durante el período de dolarización 2000 - 2016 el crecimiento promedio fue de $4,3 \%$. 


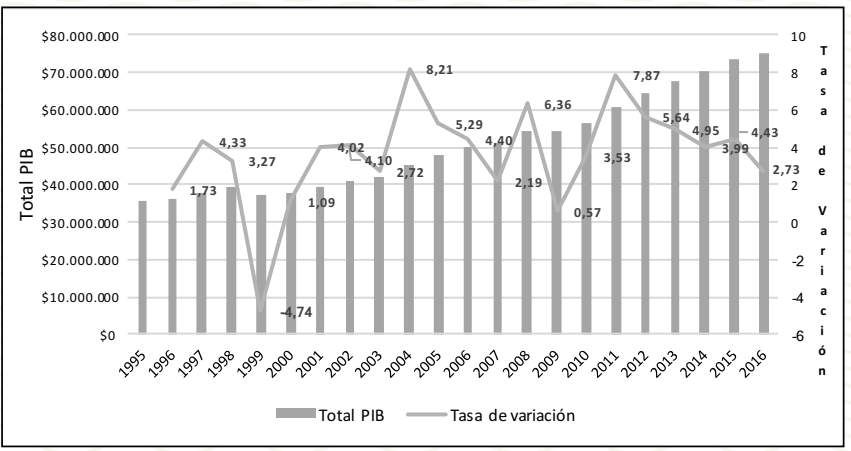

Fuente: Elaboración propia a partir del Banco Central del Ecuador Figura 1. Comportamiento de Producto Interno Bruto Periodo 1995 - 2016 (Precios de 2007

y Tasa de Variación)

La tasa de crecimiento del año 2012 fue de 5,2\%, esto debido a la continua producción petrolera, a los incrementos de la producción por parte de las empresas públicas, demanda interna, inversión en infraestructura vial, la expansión crediticia que dio lugar al incremento del consumo privado. En los años 2013 y 2014 hubo una tasa de crecimiento de $4,6 \%$ y $3,8 \%$ respectivamente, para el Banco Central del Ecuador, el sector que más ha aportado es el sector no petrolero, además dentro de las más representativas está el sector de la construcción con una demanda que incentiva su auge, también se encuentran el consumo de hogares y las exportaciones, mientras que el gasto público ha disminuido.

\section{Índice de precios al consumidor}

Durante el periodo de estudio y basado en datos que presenta el Instituto Nacional de Estadística y Censos, la inflación en el Ecuador medida a través del Índice de Precios al Consumidor para el periodo 2000 2016, ha presentado una tendencia decreciente. Se destaca también el crecimiento de los precios de consumo de la economía en el año 2000, donde la inflación alcanzó 96,1\%, este comportamiento de subida de precios se debió a una serie de factores originados por la crisis del 99, uno de ellos la rápida devaluación del sucre, y los ajustes realizados en materia de politica económica.

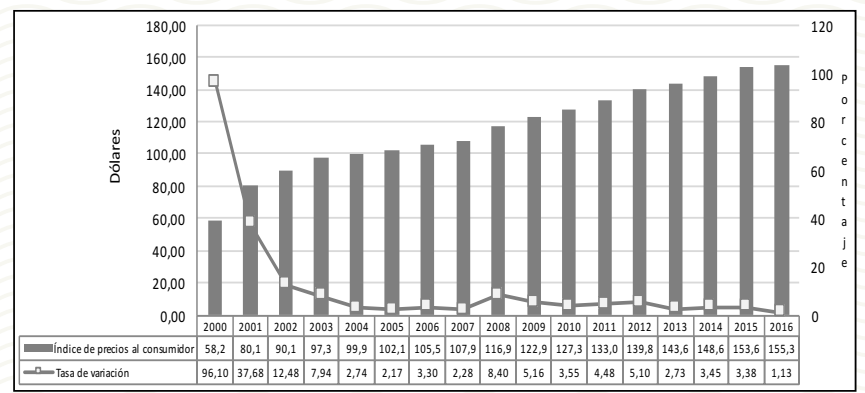

Fuente: Elaboración propia a partir del Banco Central del Ecuador Figura 2. Evolución del Índice de Precios al Consumidor Periodo 2000- 2016 (Dólares - Porcentajes)

En el año 2010 la inflación tiende a disminuir hasta un 3,55\%, tasa aún menor a la registrada en el año anterior, la misma se encontraba dentro de las proyecciones planteadas por el gobierno, donde se pronosticaba cerrar el año con 3,35\%, es decir, fue un año con estabilidad de precios. Por medio del último reporte del Índice de Precios al Consumidor que presenta el Instituto Nacional de Estadística y Censos informó que la inflación del año 2016 se ubicó en el 1,12\%, es decir, fue menor a la registrada en el 2015 que fue de 3,38\%.

\section{Gasto anual en educación}

La educación es un pilar fundamental para lograr el avance y mejoramiento del país, además es uno de los factores que más influye en el progreso de las personas permitiendo tener una sociedad con igualdad de oportunidades, mejorar sus niveles de bienestar, empleo y crecimiento económico. Según (Agencia Pública de Noticias del Ecuador y Suramérica, 2016) para el año 2014 la inversión en educación fue de \$3.395.2 millones un incremento de apenas 0,3\% al año anterior, y que fue destinado para las 25 unidades educativas del milenio que funcionaban dentro el país, mientras que otras 27 se encontraban en construcción, donde se entregó 1'405.537 uniformes a estudiantes de primaria y secundaria, 2'160.804 de alumnos recibieron 3'860.944 kits de textos y alimentación. En el año 2015 la inversión en educación pasó de 0,3\% en el 2014 a 14,8\% en el 2015.

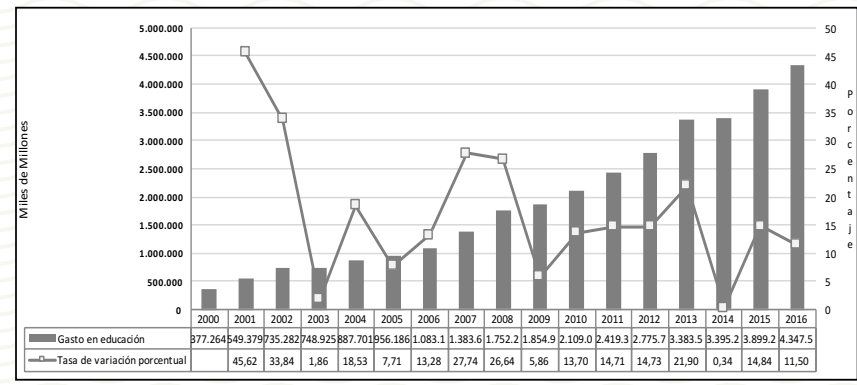

Fuente: Elaboración propia a partir del Banco Central del Ecuador Figura 3. Gasto anual en educación y variación porcentual Periodo $2000-2016$ (Miles de millones de USD - Porcentajes)

Para explicar la relación causal entre la educación, empleo y salarios, Jiménez, Jiménez, \& López, (2010), adoptaron distintos planteamientos y enfoques analíticos, llegando a la conclusión que bajo la teoría del capital humano, la demanda de educación se explica como una decisión inversora por los beneficios laborales que se derivan de las mejoras de conocimiento, por tal razón, aquellos países que han realizado mayores inversiones en educación alcanzan mayores niveles de desarrollo.

\section{Salario básico unificado}

Por medio de los datos presentados por el Banco Central del Ecuador, durante el periodo 2000-2016 el salario básico unificado ha ido incrementándose.

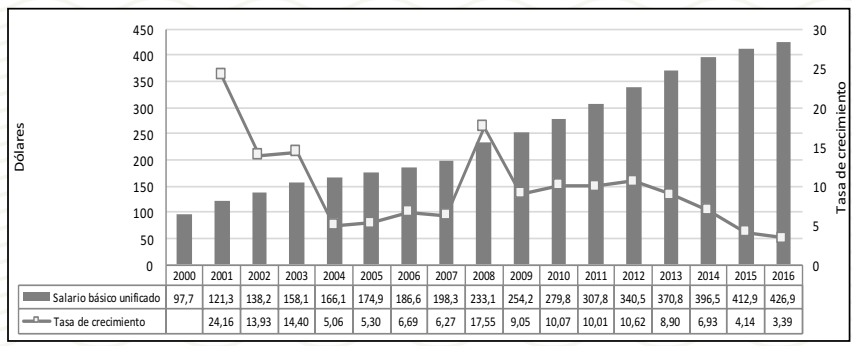

Fuente: Elaboración propia a partir del Banco Central del Ecuador Figura 4. Evolución del Salario Básico Unificado Promedio Anual. Periodo 2000 - 2016 (USD - Tasa de crecimiento)

En el año 2016 el salario básico unificado se incrementó en \$16,4, con respecto al año anterior, es decir este llegó a \$412.9. Cabe destacar que el incremento de tan solo el $4,13 \%$ se debió a la caída de los precios del crudo y a los efectos que vivió el país por la crisis económica. En lo que se refiere al año 2017, el salario básico unificado se fijó en $\$ 426,9$, es decir, hubo un incremento de $\$ 14$ dólares con respecto al 2016.

\section{Inversión en el sector eléctrico}

La energía eléctrica es fundamental para el diario vivir de las personas, ya sea para realizar sus actividades de uso doméstico, procesos industriales o tecnológicos y de comunicación. Según Guerrero (2014) en el informe sectorial "Ecuador: Sector Eléctrico" manifiesta que el país a 
través de la explotación petrolera empezó a modernizar su economía, teniendo un mayor crecimiento económico y consumo energético, así mismo manifiesta que a partir del año 2007, el Estado de turno se orientó al cambio de la matriz energética.

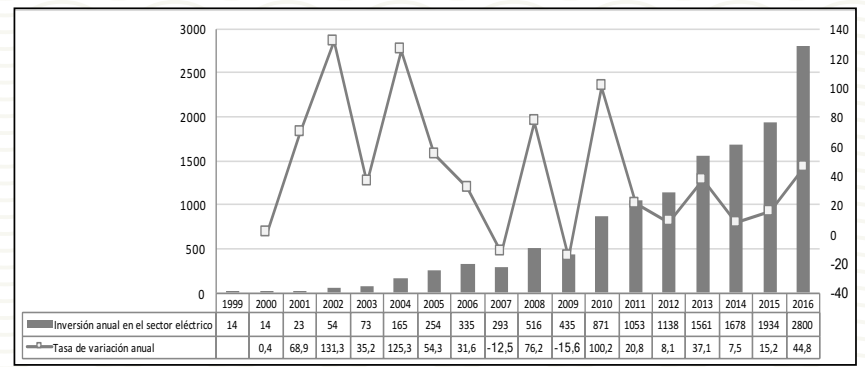

Fuente: Elaboración propia a partir del Ministerio de Electricidad y Energía Renovable Figura 5. Inversión anual en el sector eléctrico.

A través de un análisis que realiza De la Paz (2013) en el que cita el Plan Maestro de Electrificación de Ecuador 2010-2020 manifiesta que el crecimiento de la demanda de energía y de la infraestructura que la oferte, es parámetro fundamental de la potencialidad de crecimiento económico de un país. No se podrían hacer proyecciones valederas de crecimiento económico, si estas no vienen asistidas de un nivel creciente de disponibilidad de energía eléctrica, ya que la inversión productiva así lo demanda, por el contario, sin ésta se frenarían las actividades productivas.

Según (Ministerio de Electricidad y Energía Renovable, 2016)Para el año 2016 se esperaba duplicar la generación eléctrica, a través del pleno funcionamiento de los nuevos proyectos emblemáticos como son: Coca Codo Sinclair, Minas San Francisco, Delsitanisagua, Manduriacu, Mazar Dudas, Toachi Pilatón, Quijos, Sopladora y Villonaco. Para el logro de ese objetivo se invirtió $\$ 2.800$ miles de millones lo que representó un aumento del $44,8 \%$ con relación al año anterior.

\section{Inversión Extranjera Directa}

La inversión extranjera directa es importante en la economía de los países en desarrollo porque a través de ella se pueden adquirir recursos y tecnologías provenientes de grandes empresas. Por lo tanto, es necesario estudiar los volúmenes que el país ha captado, para lo cual se considera los datos obtenidos del Banco Central del Ecuador.

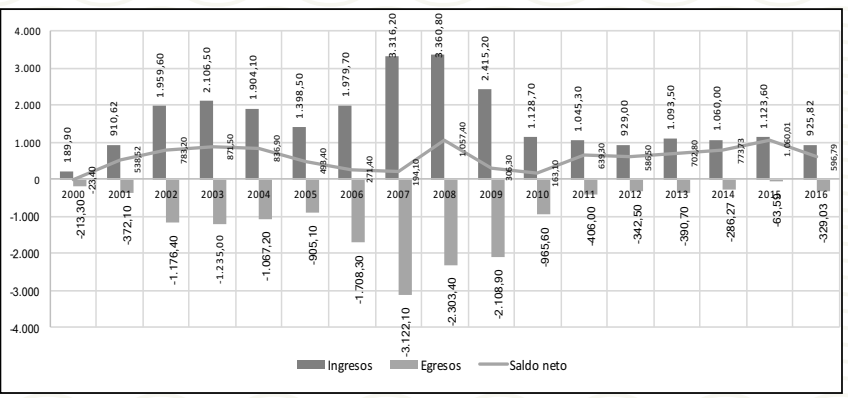

Fuente: Elaboración propia a partir del Banco Central del Ecuador Figura 6. Inversión Extranjera Directa Periodo 2000 - 2016 (Millones de USD)

Durante el año 2014 la Inversión Extranjera Directa incrementó en un $10 \%$ con un monto de $\$ 773,73$ millones de dólares. En el 2015 , de acuerdo a la información estadística proporcionada por la Comisión Económica para América Latina y el Caribe, en Ecuador se registró un récord histórico en las entradas de IED, con un aumento del 37\% con relación al año anterior, la IED neta alcanzó los \$1060 millones, de los cuales estuvo destinado al sector petrolero (32\%), y a la industria manufacturera (24\%). Por último en el año 2016 la IED nuevamente disminuye llegando a $\$ 596,79$ millones, los países q más ingresos reportaron fueron Estados Unidos con 18\%, seguido de Perú y China, con $16 \%$ y $9 \%$ respectivamente.
Estimación del modelo: Variables del modelo

Tabla 1. Estimación de los parámetros del modelo econométrico

Method: Least Squares

Sample: 2000Q1 2016Q4
Included observations: 60

\begin{tabular}{|c|c|c|c|c|}
\hline Variable & Coefficient & Std Error & t-Statistic & Prob. \\
\hline $\mathrm{C}$ & 0.037261 & 5.791952 & 0.006433 & 0.9949 \\
\hline LOGX & 0.105475 & 0.431652 & 0.244352 & 0.0080 \\
\hline LOGX1 & 2.669505 & 1.451713 & 1.838866 & 0.0724 \\
\hline LOGX2 & 2.315952 & 1.881122 & 1.231155 & 0.2245 \\
\hline LOGX3 & 0.317899 & 1.221650 & 0.260221 & 0.0559 \\
\hline LOGX4 & 8.710582 & 3.316401 & -2626517 & 0.0117 \\
\hline R-squared & 0.516664 & \multicolumn{2}{|c|}{ Mean dependent var } & 5.032088 \\
\hline Adjusted R-squared & 0.464127 & \multirow{2}{*}{\multicolumn{2}{|c|}{$\begin{array}{l}\text { S.D. dependent var } \\
\text { Akaike info criterion }\end{array}$}} & 0.718214 \\
\hline S.E. of regression & 0.525756 & & & 1.660210 \\
\hline Sum squared resid & 1271531 & & 1.885353 \\
\hline Log likelihood & -3716545 & \multirow{2}{*}{\multicolumn{2}{|c|}{$\begin{array}{l}\text { Hannan-Quinn criter } \\
\text { Durbin-Watson stat }\end{array}$}} & 1.746524 \\
\hline F-statistic & 9834360 & & & 2.039536 \\
\hline Prob (F-statistic) & 002 & & & \\
\hline
\end{tabular}

Donde, se expresa la siguiente ecuación:

LOGIED $=0.0372+0.1054^{*}$ LOGELEC $+2.6695^{*}$ LOGIPC + $2.3159^{*}$ LOGSBU + 0.3178*LOGEDU + 8.7105*LOGPIB

$\beta 0=0.0372$, el valor del intercepto de alrededor de 60 , significa que si valores de las variables explicativas fuesen cero, la inversión extranjera directa promedio se incrementaría en un 0,03\%.

$\beta 1=0.1054$, es el coeficiente de regresión parcial de la inversión eléctrica e indica que, si se mantiene constante la influencia de IPC, SBU, EDU y PIB; conforme aumenta en 1\% la inversión eléctrica en el país, estimula un incremento de $0.10 \%$ en la inversión extranjera directa.

$\boldsymbol{\beta} 2=2.6695$, es el coeficiente de regresión parcial del índice de precios al consumidor, lo cual significa que al mantener constante o cero, la influencia de ELEC, SBU, EDU y PIB; la IED se incrementará en un $2.67 \%$, ante un incremento de $1 \%$ en el IPC.

$\beta 3=2.3159$, representa al coeficiente parcial respecto al SBU, el cual, significa que al mantener constante la influencia de ELEC, IPC, EDU y PIB; un incremento de $1 \%$ en el salario básico unificado, provoca un acrecentamiento de $2.31 \%$ en la inversión extranjera directa, expresando un relación directa.

$\beta 4=0.3178$, corresponde al coeficiente de regresión parcial de la inversión en educación e indica que, si se mantiene constante la influencia de ELEC, IPC, SBU y PIB real; un aumento de 1\% en la inversión en educación, provoca un incremento de $0.32 \%$ en la inversión extranjera directa, expresando una relación directa.

$\beta 5=8.7105$, es el coeficiente de regresión parcial de PIB, y que significa que al mantener constante la influencia de ELEC, IPC, SBU y EDU; un aumento de $1 \%$ en el PIB real, incita un incremento de $8.71 \%$ en la IED; ejerciendo la relación directa sobre la inversión directa.

$\mathbf{R} 2=0.5166$, mide la bondad de ajuste de la ecuación de regresión; es decir, el porcentaje de la variación total en la variable dependiente $Y$ explicada por las variables explicativas $X$. El valor de R2 de 0.51 significa que el $51 \%$ de la variación en la inversión extranjera directa se explica mediante ELEC, IPC, SBU, EDU y PIB.

\section{Conclusiones}

La dolarización ha contribuido positivamente al desempeño de la economía, confiriéndole estabilidad macroeconómica, a la vez logró reducir la inflación a niveles que bordean el $2 \%$ en promedio para el periodo de estudio.

La inversión extranjera directa en los últimos 4 años ha logrado mantenerse en alrededor de $\$ 675,58$ millones en promedio anual, los de- 
terminantes que más influyeron son los relacionados con el tamaño del mercado, la estabilidad económica, el capital humano y los costos de producción e infraestructura.

Los resultados obtenidos de la estimación econométrica demuestran que la dolarización, medida a través de los indicadores: Inversión en electricidad (0.1054), índice de precios al consumidor (2.6695), salario básico unificado (2.3159), gasto en educación (0.3178) y PIB (8.7105) tienen una relación directa con la inversión extranjera directa.

\section{Referencias}

Acosta, A. (18 de Febrero de 2010). Zona económica. Obtenido de http://www.zonaeconomica.com/ecuador/evolucion-inflacion/ inflacion

Bilbao, J., \& Longás, J. (2009). Temas de Economía Mundial. Madrid: Rumagraf.

De la Paz, M. (2013). Plan Maestro de Electrificación 20102020. Gestión, 38.

Emanuel, C. (21 de Julio de 2015). Repositorio de la Universidad de Especialidades Espiritu Santo. Obtenido de http://www.uees. edu.ec/dolarizacion/pdf/3/6-Conf-Carlos-Julio-Emmanuel-210115.pdf

Guerrero, A. (2014). Informe Sectorial Ecuador: Sector Eléctrico. 30 de noviembre de 2014. Quito: ratingspcr.

Jiménez, J., Jiménez, M., \& López, M. (17 de Marzo de 2010).
Comisión Económica para América Latina y el Caribe. Obtenido de http://www.cepal.org/ilpes/noticias/paginas/1/41751/salinas_rahona_salinas_doc.pdf

Larrea, C. (2008). Pobreza, dolarización y crisis en el Ecuador. Quito: Abya-Yala.

Ponce, J. (2010). Políticas educativas y desempeño: una evaluación de impacto de programas educativos. Quito: Rispergraf.

Rosillo. (26 de Octubre de 2011). Universidad Politécnica Salesiana Sede Quito. Obtenido de http://dspace. ups.edu.ec/bitstream/123456789/4452/1/UPS-QT00055.pdf Banco Central del Ecuador. (07 de 10 de 2016). Banco Central del Ecuador. Obtenido de https://contenido.bce.fin.ec/documentos/PublicacionesNotas/Notas/Dolarizacion/dolarizar.html Comisión Económica para América Latina y el Caribe. Recuperado de $h$ ttp://www.cepal.org/es

Instituto Nacional de Estadísticas y Censos (INEC). Recuperado de http://www.ecuadorencifras.gob.ec/institucional/home/ Ministerio de Educación. Recuperado de https://educacion.gob.ec/ Ministerio de Electricidad y Energía Renovable. Recuperado de http://www.energia.gob.ec/

Secretaría Nacional de Educación Superior, Ciencia y Tecnología. Recuperado de http://www.ces.gob.ec/index.php?option=com_sobipro\&pid=390\&sid=386:Eco-Rene-Ramirez-Gallegos\&/temid $=424$ 